\title{
SITUACIÓN ACTUAL DE LA RTU DE PRÓSTATA EN PACIENTES CON PSA ELEVADO
}

\author{
Koenraad M.L.E. van Renterghem?', Philip E.V.A. van Kerrebroeck ${ }^{2}$ y Gommert A.Van \\ Koeveringe ${ }^{2}$.
}

'Servicio de Urología Virga Jesse Hospital. Hasselt. Bélgica.

2Servicio de Urología. Academic Hospital Maastricht. Holanda.

Resumen.- OBJETIVO: El objetivo de esta revisión fue evaluar el papel de la resección transuretral de próstata (RTUP) en pacientes con PSA elevado. Se analizaron los resultados tanto en pacientes sintomáticos como asintomáticos.

MÉTODOS: Se revisó la literatura actual (1987-2009) en relación con esta población específica de pacientes. Esta investigación se realizó mediante búsqueda en Medline.

RESULTADOS: Inicialmente, estrategias terapéuticas distintas a la RTUp suelen ser utilizadas en pacientes con PSA elevado y sin evidencia de CP (CP). Este trabajo discute el valor de la RTUp en pacientes con PSA eleva-

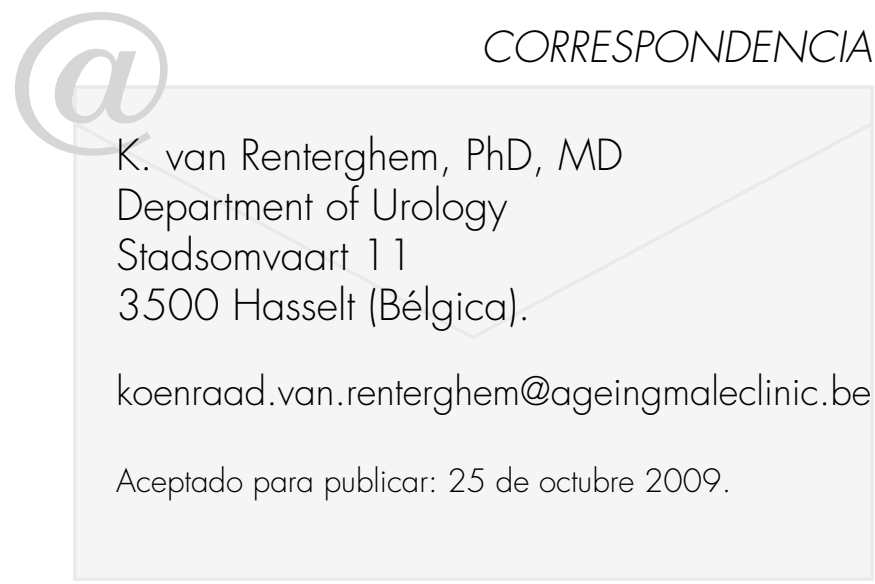

do o incremento progresivo del PSA. En este contexto, la RTUPp se puede realizar con o sin biopsia prostática concomitante. Además, la RTUp se puede proponer a pacientes con y sin STUI.

CONCLUSIONES: En esta revisión, se evaluó el papel de la RTUp en pacientes con PSA elevado o incremento progresivo del PSA sin diagnóstico de CP. Creemos que la RTUp tiene un lugar en esta población de pacientes, incluso en aquellos que no presenten STUI. Esta estrategia está bien fundamentada después de una revisión extensa de la literatura disponible. En la mayoría de los casos, una RTUp se traducirá en una normalización del PSA, así como un beneficio sintomático. Además, este procedimiento permitirá una extensa evaluación histológica, que podría aportar un potencial diagnóstico incidental de CP.

Palabras clave: Cáncer de próstata. Resección transuretral de próstata. PSA. Obstrucción del tracto urinario inferior. Síntomas del tracto urinario inferior. Biopsia de próstata.

\section{Lista de abreviaturas}

BOO: Obstrucción Tracto Urinario Inferior HBP: Hiperplasia Benigna de la Próstata STUI: Síntomas del Tracto Urinario Inferior PSA: Antígeno Específico de la Próstata RTUp: Resección Transuretral de Próstata $C P: C P$

Summary.- OBJECTIVES: The aim of this review is to evaluate the place of transurethral resection of the prostate (TURP) in patients with elevated and/or rising prostate specific antigen (PSA). The role of TURP in these patients 
is assessed in symptomatic as well as in asymptomatic patients.

METHODS: Current literature (1987-2009) was reviewed with regard to this specific population. This research was performed using the Medline online search tools.

RESULTS: Initially, possible therapeutic strategies other than TURP could be used in patients with elevated and/ or rising PSA values and no sign of proven prostate cancer. Consequently, the value of TURP in patients with elevated and/or rising PSA and no proven prostate cancer is discussed. In this setting, TURP can be executed with or without concomitant prostate biopsies. Furthermore, TURP can be proposed to patients with and without lower urinary tract symptoms.

CONCLUSIONS: In this review, we evaluated the role of TURP in patients with elevated and/or rising PSA values and no proven prostate cancer. We believe TURP has a place in this particular population even in patients without lower urinary tract symptoms. This strategy is well founded on solid ground after an extensive review of the available literature. In most cases, a TURP will result in a normalization of PSA as well as a symptomatic benefit. Additionally, this procedure will allow histological evaluation which might show a possible life threatening prostate cancer in some patients.

Keywords: Prostate cancer. Transurethral resection of the prostate. Prostate specific antigen. Bladder outlet obstruction. Lower urinary tract symptoms. Prostate biopsy.

\section{INTRODUCCIÓN}

Con la introducción del PSA, se dispuso de una valiosa herramienta en el estudio diagnóstico de los pacientes con patología prostática (1). En la práctica urológica, los pacientes con un PSA elevado y / o aumento del valor del PSA son una población creciente. Aunque el PSA se clasifica como un marcador tumoral, no es cáncer específico $(2,3)$. Esto explica el hallazgo de valores elevados de PSA en pacientes en los que no se ha encontrado CP. La incertidumbre es aún mayor, si el PSA sigue aumentando y no se puede encontrar el tumor, (4-6). Hasta ahora, se han publicado muchos artículos proponiendo cómo tratar a estos pacientes. Algunos autores han sugerido tratar a los pacientes con antibióticos y / o aproximaciones dietéticas, hasta ahora, sin un fundamento científico sólido (7-12). Otros han sugerido refinar el diagnóstico con derivados del PSA, como PSA libre / total, densidad de PSA, velocidad de PSA, BPSA, PSA ajustado según la edad, o PSA complejo (13-24). Además, se han realizado varios intentos para aumentar el rendimiento de la biopsia ampliando el número de cilindros, cambiando zonas prostáticas que podría ser biopsiadas o repitiendo la biopsia (25-31). Además, se han desarrollado nuevas técnicas de imagen en un intento de aumentar el diagnóstico de CP. Las técnicas que ofrecen esta perspectiva, son la ecografía con contraste, la elastografía en tiempo real, la espectroscopia asociada a la resonancia magnética mejorada con contraste dinámico,el histoescaner, etc (32-38). Sin embargo, se necesitan más pruebas para confirmar la validez de estas nuevas técnicas. Por último, pero no menos importante, se han desarrollado los nuevos marcadores moleculares, como el PCA3 (39-40).

Sin embargo, persiste un número importante de pacientes en los que no se ha encontrado ninguna explicación para la elevación y / o el aumento de los niveles de PSA. En los últimos años, se han acumulado mas evidencias del papel beneficioso de la RTUp en esta población de pacientes. En esta revisión, se evaluará el papel de la RTUp y otras estrategias terapéuticas en esta población de pacientes en particular.

\section{MATERIAL Y MÉTODOS}

Se revisó la literatura actual con respecto a esta población específica. Esta investigación se realizó utilizando las herramientas a disposición del público mediante búsqueda en Medline. Se revisó la literatura desde 1987 hasta 2009. Se utilizaron los siguientes términos de búsqueda: "RTUp diagnósti-

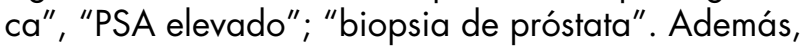
se realizó la búsqueda de referencias posteriores en los artículos recuperados.

\section{RESULTADOS}

\section{Posibles estrategias terapéuticas distintas de la RTUp para tratar a los pacientes con un elevado y / o au- mento del valor del PSA y CP no probado.}

Dado que es muy frecuente tratar mediante tratamiento médico a pacientes con hiperplasia benigna de la próstata (HBP), se han desarrollado numerosas moléculas para el tratamiento específico de esta población de pacientes. Uno podría preguntarse si el tratamiento médico no sería una opción terapéutica para pacientes con PSA elevado y / o aumento del valor de PSA y el CP no probado. Durante años, se han utilizado los $\alpha$-antagonista adrenérgicos $\alpha-1$. Estos medicamentos pueden ser muy eficaces para el tra- 
tamiento de los STUI. Desgraciadamente, no ejercen ningún efecto sobre los valores del PSA (41). Por otro lado, se sabe que los inhibidores de la 5 areductasa (5ARI) como el finasteride y el dutasteride tienen efecto sobre los valores del PSA. En la mayoría de los casos, la "regla de multiplicar por dos "se propone para calcular el valor real de PSA en pacientes tratados con 5ARI. Sin embargo, Brawer demostró que "la regla de multiplicar por dos "no siempre es correcta (41). Pero en general se acepta esta regla como útil y práctica. Por otra parte, debe tenerse en cuenta que un valor elevado de PSA no puede ser la única indicación para iniciar tratamiento con un 5ARI. Esto no cambia el hecho de que el valor de PSA este elevado, cualquiera que sea la técnica utilizada para evaluar el "verdadero" valor de PSA (42-47). Muchos urólogos tratan de dar a estos pacientes un tratamiento empírico con antibióticos. Sin embargo, en la literatura no hay pruebas de que este tratamiento normalice el valor de PSA. Por otra parte, hasta el momento no se ha demostrado que la inflamación crónica de la próstata pueda influir en los valores de PSA $(12,48-51)$. Hasta hace poco se creía que la manipulación de la dieta era una estrategia eficaz en esta población de pacientes. Hasta este momento, falta evidencia científica para apoyar esta hipótesis. Hasta la fecha, es absolutamente prematuro, abogar por la manipulación de la dieta para normalizar los valores de PSA o incluso para reducir el riesgo de CP (7-11).

\section{Valor de la RTUp de próstata en pacientes con PSA elevado y / o aumento de los valo- res del PSA}

\section{El valor de la RTUp con biopsia de próstata concomi- tante en pacientes con PSA elevado y / o aumento de los valores del PSA.}

En 1997, Ornstein describió el impacto de la biopsia de próstata en la incidencia de CP en varones con STUI que se sometieron a una RTUp (52). En este análisis retrospectivo de 58 pacientes consecutivos, Ornstein mostró que los CP relevantes pueden ser detectados en más del 15\% de los pacientes sometidos a RTUp, por razones sintomáticas. En esta población, los pacientes no tenían síntomas de CP y tuvieron una o más biopsias de próstata sistemáticas negativas. Puppo evaluó el mismo procedimiento en 43 pacientes (53). Fue capaz de realizar una RTUp de próstata en 14 pacientes, en este grupo de 43. A estos pacientes se les ofreció una RTUp y la obstrucción del tracto urinario inferior (BOO) no tuvo ninguna influencia en la decisión. En este pequeño grupo de pacientes, Puppo concluyó que la RTUp combinada con biopsia transrectal de próstata en el mismo acto tiene un alto rendimiento diagnóstico.
Resultado de la RTUp en pacientes con PSA elevado y / o aumento del PSA y STUI, sin signos de CP demostrado.

En pacientes con elevado y / o aumento del PSA con STUI, los valores del PSA se correlacionan con el crecimiento futuro de la próstata, mayor riesgo de retención urinaria aguda, obstrucción del tracto urinario inferior, y una mayor necesidad de futuro tratamiento quirúrgico para aliviarlos STUI (54-59). Por otra parte, la RTUp es un tratamiento adecuado para aliviar STUI debido a obstrucción del tracto urinario inferior (60). Además, la RTUp se traducirá en un beneficio sintomático y permitirá a un examen histológico extenso.

Aunque el número de cánceres de próstata detectados por RTUp se redujo después de la introducción del PSA, se sabe que el CP puede ser detectado por RTUp en pacientes sin sospecha de CP. Merril mostró que en la era del PSA los casos de cánceres detectados por la RTUp representaban aproximadamente el 10\% de todos los casos de CP detectados (61). Varios autores han tratado de mejorar el diagnóstico de $\mathrm{CP}$ en pacientes con biopsias prostáticas negativas previas, mediante la realización de biopsia transuretral de la próstata. Sin embargo, sabiendo que la mayoría de los CP se presentan en la zona periférica, una biopsia transuretral de la próstata no es una buena estrategia para diagnosticar $\mathrm{CP}$ en pacientes con biopsias de próstata previamente (62-64) negativas.

Kitamura fue el primero en describir los resultados de una RTUp en pacientes con biopsias previas negativas (65). En este análisis prospectivo, se incluyeron 139 pacientes consecutivos. Estos pacientes fueron sometidos a RTUp después de biopsias negativas, cuando el tratamiento de los síntomas así lo hizo necesario. Kitamura concluyó que no estaba claro si la RTUp es una buena opción para el diagnostico de cáncer en esta población de pacientes. Radhakrishnan presentó sus resultados en un análisis retrospectivo de un pequeño grupo de 14 pacientes (66). En este grupo, 3 pacientes tenían un CP que potencialmente ponía su vida en peligro y que fue detectada mediante RTUp. Radhakrishnan informó que la RTUp se puede aplicar en esta población de pacientes.

Zigeuner presentó la tasa de detección del CP mediante RTUp o adenomectomía abierta en un gran estudio retrospectivo de pacientes $(1189$ pacientes consecutivos entre 1994 y 2000), que previamente había tenido resultados negativos en biopsias transrectales de próstata (67). Todos los pacientes fueron tratados por obstrucción del tracto urinario inferior 
sintomática. Zigeuner encontró una tasa global de $\mathrm{CP}$ del $7,9 \%$. Si los pacientes tenían un tacto rectal normal, la incidencia de CP era de 5,5\%. Philip presentó los resultados sobre la importancia de la RTUp en pacientes con antecedentes de múltiples biopsias negativas de próstata (68). En este documento, se muestra la importancia de una resección anterior de la próstata con respecto al diagnóstico de CP.

\section{La importancia de la RTUp de próstata en pacientes con elevado y / o PSA en aumento, sin signos de CP demostrado y $\sin$ STUI.}

La incertidumbre aumenta cuando un paciente presenta un PSA elevado y / o PSA en aumento sin datos de CP en el tacto rectal, ecografía transrectal y (múltiples) biopsias de próstata y sin STUI. En esta población en particular, se puede esperar que se produzca obstrucción del tracto urinario inferior (69). Dado que una obstrucción del tracto urinario inferior no tratada puede llevar a situaciones graves como la hipertrofía del detrusor y a insuficiencia renal, puede ser un buen motivo para plantear una RTUp (70-71). En esta población, realizando una RTUp se producirá un beneficio sintomático, una normalización del valor del PSA y además este procedimiento permite un amplio examen histológico. En la mayoría de los casos, este examen histológico muestra que no hay $C P$, aunque se encuentra $C P$ en algunos pacientes. En un análisis retrospectivo, se encontró $\mathrm{CP}$ en el 9,8\% de los pacientes que, en la mayoría de los casos, necesitaban un tratamiento adicional (72-73). Sorprendentemente, cuando se realizó una prostatectomía radical, se encontro casi siempre CP residual localizado en la zona periférica (anterior y lateral) de la próstata. En un análisis prospectivo, nos encontramos con "HBP pura" en el $81,8 \%$ de los casos. En el $12,1 \%$ de los pacientes, se encontró un $C P$ insignificante, mientras que el $C P$ agresivo que requirió tratamiento adicional fué hallado en el 6,1\% de los pacientes (74). Una vez más, el tumor residual se encontró en la zona periférica anterior y lateral de la próstata.

En esta población de este estudio específico, se mostró que los resultados a largo plazo de este procedimiento fueron excelentes. Sin embargo, se recomienda un control anual de PSA, ya que en el $2,8 \%$ de estos pacientes hubo recidiva bioquímica después de varios años, lo que requirió tratamiento adicional. Como propuesta para incluir en un nuevo algoritmo, hemos sugerido un papel clave para el estudio urodinámico en el seguimiento de esta población de pacientes ya que es de esperar que se produzca a medio plazo obstrucción del tracto urinario inferior (75).

\section{DISCUSIÓN}

Nuestra sociedad occidental se caracteriza por un envejecimiento de la población cada vez mayor debido a la mejoría de la salud en general que lleva a una esperanza de vida cada vez mayor. Esto significa que la HBP y el CP son más frecuentes, ya que son enfermedades del varón que envejece. Más y más hombres alcazanzaran la edad suficiente para tener una "enfermedad" prostática. Además, los hombres son cada vez más conscientes de las enfermedades de la próstata y son conscientes de la existencia del PSA. Por otra parte, el potencial efecto letal del $\mathrm{CP}$ es bien conocido y nos lleva a la aparición de nuevas "enfermedades", tales como "la ansiedad del PSA" (6).

Por esta razón, los hombres con PSA elevado y / o aumento de los niveles de PSA consultan con un urólogo. Cuando la elevación del PSA se correlaciona con un examen digital rectal sospechoso, una ecografía transrectal sospechosa y amplias biopsias de próstata positivas, en la mayoría de los casos se sabe cual es el paso siguiente Sin embargo, cuando el PSA es elevado y sigue aumentando en pacientes con "múltiples" biopsias prostáticas negativas, incluso en las biopsias de próstata por saturación, la incertidumbre aumenta, no sólo para el paciente y el médico de atención primaria, sino también para el urólogo. Como sabemos, la repetición de biopsias por saturación no es una opción adecuada en estos pacientes ya que la posibilidad de encontrar CP con relevancia clínica se reduce con cada nueva biopsia de próstata realizada (30-31). El tratamiento con antibióticos, la manipulación dietética, los 5ARI y $\alpha$ -bloqueantes no son una buena solución en esta población (7-11, 41-51).

Si los pacientes estan sintomáticos, podría estar indicada una RTUp, incluso sin realizar una evaluación urodinámica previa. Creemos que una serie de biopsias de próstata amplias es suficiente en esta población (70). En este grupo, la RTUp puede ser considerada no sólo como un procedimiento terapéutico, sino también como un procedimiento de diagnóstico ya que el CP se encuentra en gran cantidad de pacientes. Creemos que la RTUp concomitante con la biopsia tiene un rendimiento relativamente pobre para diagnosticar cáncer, aunque se necesitan más investigaciones con biopsias que incluyan un mayor número de cilindros ( 21 cilindros?) (52-53).

El grupo más difícil son los pacientes sin "STUI molestos". En esta población, se debe realizar un estudio urodinámico después de al menos 2 biopsias de próstata por saturación (75). Una evaluación urodinámica en este grupo de pacientes puede 
demostrar obstrucción del tracto urinario inferior en casi todos los casos para los que se puede proponer una RTUp $(69,72-75)$. Esta exploración urodinámica tiene un gran valor predictivo en el resultado de una RTUp en pacientes con obstrucción del tracto urinario inferior (76-79). La RTUp se traducirá en el tratamiento de la obstrucción del tracto urinario inferior, la supernormalización del PSA, el beneficio sintomático y examen histológico extenso. Si se encuentra un $C P$ insignificante, se puede proponer al paciente una estrategia de vigilancia activa. Sin embargo, si se encuentra CP agresivo y potencialmente mortal, es la cirugía radical la que debe ser propuesta a estos pacientes. Colombo et al. demostraron que en manos muy expertas, se puede realizar una prostatectomía radical con buenos resultados después de una RTUp previa (80).

En conclusión, hay varios argumentos a favor de proponer una RTUp a estos pacientes. En primer lugar, las obstrucciones del tracto urinario inferior, sintomáticas o no, deben ser tratadas. La obstrucción del tracto urinario inferior no tratada puede llevar a situaciones graves como la descompensación del detrusor y la insuficiencia renal (70-71).

Además, el nivel de PSA postoperatorio se reducirá en la mayoría de los casos de manera significativa. Por otra parte, es posible un amplio examen histológico. Si solo se encuentra HBP, los pacientes pueden estar tranquilos. Si se encuentra CP agresivo y potencialmente mortal, se debe proponer cirugía radical a los pacientes para tratar un fumor que de otro modo se diagnosticaría en una etapa posterior y menos curable. Si, por otra parte, se encuentra un $\mathrm{CP}$ insignificante, se debe tratar con el paciente una estrategia de estrecha vigilancia. Dado que los niveles de PSA se relacionan con la obstrucción del tracto urinario inferior, podemos concluir que el nivel elevado de PSA no sólo está relacionado con la presencia de $C P$, sino también con la obstrucción del tracto urinario inferior.

Hoy en día se puede considerar que es un riesgo no el sobrediagnóstico sino el sobretratamiento, los pacientes se pueden beneficiar de una eventual cirugía radical. Esto implica que, en este grupo, las técnicas de vaporización de la próstata con láser no deben ser defendidas. Por último y no menos importante, tambíen deben tenerse en cuenta los argumentos económicos. Se sabe que el tratamiento médico para una enfermedad progresiva, de larga duración en una población que envejece es mucho más costoso y no proporciona la curación de la enfermedad (81-84). Además, no hay ningún efecto real en el nivel de PSA en la mayoría de los casos y no hay posibilidad de un examen histológico para excluir un
CP. Por lo tanto, se debe ofrecer a los pacientes una RTUp. Es un tratamiento con excelentes y probados resultados a largo plazo. Además, debido a la mejora de las técnicas quirúrgicas, la RTUp no debe ser considerada como un procedimiento "invasivo" (8589).

\section{CONCLUSIÓN}

Los pacientes con PSA elevado y / aumento de los niveles de PSA y sin signos de CP en el tacto rectal, ecografía transrectal o después de múltiples biopsias de próstata, constituyen una población importante en la práctica urológica. Si los pacientes presentan STUI, se puede proponer la realización de una RTUp, ya que ello resultará un beneficio sintomático, normalización de los niveles de PSA y la posibilidad de un examen histológico más extenso. Sin embargo, si los pacientes no tienen STUI, se debe realizar un estudio urodinámico después de al menos dos biopsias prostáticas por saturación. Es posible encontrar datos de obstrucción del tracto urinario inferior en estudios de presión-flujo. Esta obstrucción del tracto urinario inferior puede ser tratada mediante RTUp, ya que la obstrucción sin tratar puede tener riesgos potenciales. Incluso en esta población se verá un beneficio sintomático, supernormalización del PSA y tendremos la posibilidad de un examen histológico más extenso.

Por esta razón, la RTUp no sólo debe considerarse como un procedimiento terapéutico, sino también como un procedimiento diagnóstico. En la mayoría de los casos sólo se encuentra HBP, en un número de casos un $\mathrm{CP}$ insignificante, $\mathrm{y}$ en otros un agresivo y amenazante $C P$.

\section{AGRADECIMIENTOS}

Los autores agradecen al Dr. S. Deferme (Pharma XL, Bélgica) por su ayuda en la escritura y la publicación de este manuscrito.

\section{BIBLIOGRAFIA y LECTURAS RECOMENDADAS ( ${ }^{*}$ lectura de interés $y^{* *}$ lectura fundamental)}

1. Stamey T, Yang N, Hay A, McNeal J, Freiha F, Redwine E. Prostate-specific antigen as a serum marker for adenocarcinoma of the prostate. $\mathrm{N}$ Engl J Med. 1987;317:909-16.

2. Laguna P, Alivizatos G. Prostate specific antigen and benign prostatic hyperplasia. Curr Opin Urol. 2000;10:3-8. 
3. Stephan C, Jung K, Lein M, Diamandis E. PSA and other tissue kallikreins for prostate cancer detection. Eur J Cancer. 2007;43:1918-1926.

4. Katz D, Jarrard D, McHorney C, Hillis S, Wiebe D, Fryback D. Health perceptions in patients who undergo screening and workup for prostate cancer. Urology 2007;69:215-220.

5. Roth A, Christian N, Rosenfeld B, Warshowski A, O'Shea N, Scher H, et al. Assessing anxiety in men with prostate cancer: further data on the reliability and validity of the Memorial Anxiety Scale for Prostate Cancer (MAX-PC). Psychosomatics 2006;47:340-347.

6. Lofters A, Juffs H, Pond G, Tannock I. "PSA-it is": knowledge of serum prostate specific antigen and other causes of anxiety in men with metastatic prostate cancer. J Urol. 2002;168:2516-2520.

7. Meyer F, Galan P, Douville P, Bairati I, Kegle P, Bertrais $\mathrm{S}$, et al. Antioxidant vitamin and mineral supplementation and prostate cancer prevention in the SU.VI.MAX trial. Int J Cancer. 2005;116:182186.

8. Kirsh V, Mayne S, Peters U, Chatterjee N, Leitzmann M, Dixon B, et al. A prospective study of lycopene and tomato product intake and risk of prostate cancer. Cancer Epidemiol Biomarkers Prev. 2006;15:92-98.

9. Demark-Wahnefried W, Moyad M. Dietary intervention in the management of prostate cancer. Curr Opin Urol. 2007;17:168-170.

10. Jatoi A, Burch P, Hillman D, Vanyo J, Dakhil S, Nikcevich D, et al. A tomato-based, lycopenecontaining intervention for androgen-independent prostate cancer: results of a phase II study from the North Central Cancer Treatment Group. Urology. 2007;36:289-294.

11. Eastham J, Riedel E, Latkany L, Fleisher M, Schatzkin A, Lanza E, et al. Dietary manipulation, ethnicity and serum PSA levels. Urology. 2003;62:677-685.

12. Kobayashi M, Nudui A, Morita T. Serum PSA and percent free PSA value changes after antibiotic treatment. A diagnostic method in prostate cancer suspects with asymptomatic protatitis. Urol Int. 2008;80:186-192.

13. Catalona W, Smith D, Wolfert R, Wang T, Rittenhouse H, Ratliff T, et al. Evaluation of percentage of free serum prostate-specific antigen to improve specificity of prostate cancer screening. JAMA. 1995;274:1214-1220.

14. Benson MC, Whang IS, Olsson CA, McMahon DJ, Cooner WH. The use of prostate-specific antigen density to enhance the predictive value of intermediate levels of serum prostate-specific antigen. J Urol. 1992;147:817-821.

15. Catalona W, Southwick P, Slawin K, Partin A, Brawer M, Flanigan R, et al. Comparison of per- cent free PSA, PSA density, and age-specific PSA cut-offs for prostate cancer detection and staging. Urology. 2000;56:255-260.

16. Carter HB, Pearson JD, Metter JE, Brant LJ, Chan DW, Andres R, et al. Longitudinal evaluation of prostate-specific antigen levels in men without prostate disease. JAMA. 1992:267;2215-2220.

*17. Loeb S, Roehl KA, Catalona WJ, Nadler RB. Prostate specific antigen velocity threshold for predicting prostate cancer in young men. J Urol. 2007;177;899-902.

18. Berger A, Deibl M, Strasak A, Bektic J, Pelzer A, Klocker H, et al. Large-scale study of clinical impact of PSA velocity: long-term PSA kinetics as method of differentiating men with from those without prostate cancer. Urology. 2007;69:134138.

*19. Connolly D, Black A, Murray L, Napolitano G, Gavin A, Keane P. Methods of calculatine prostatespecific antigen velocity. Eur Urol. 2007;52:10441051.

20. Khan MA, Sokoll LJ, Chan DW, Mangold LA, Mohr P, Mikolajczyk SD, et al. Clinical utility of proPSA and "benign" PSA when percent free PSA is less than 15\%. Urology. 2004;64:1160-1164.

21. Slawin KM, Shariat S, Canto E. BPSA: a novel serum marker for benign prostatic hyperplasia. Rev Urol. 2005;7:S52-S56.

22. Linton HJ, Marks LS, Millar LS, Knott CL, Rittenhouse HG, Mikolajczyk SD. Benign prostaticspecific antigen (BPSA) in serum is increased in benign prostate disease. Clin Chem. 2003;49:253259.

23. Froehner M, Hakenberg OW, Schmidt KR, Meye A, Wirth MP. Comparison of the clinical value of complexed PSA and total PSA in the discrimination between benign prostatic hyperplasia and prostate cancer. Urol Int. 2006;76:27-30.

24. Borer JG, Sherman J, Solomon MC, Plawker MW and Macchia RJ. Age specific prostate specific antigen reference ranges: population specific. $\mathbf{J}$ Urol. 1998;159:444-448.

25. Durkan GC, Sheikh N, Johnson P, Hildreth AJ, Greene DR. Improving prostate cancer detection with an extended-core transrectal ultrasonography-guided prostate biopsy protocol. BJU Int. 2002;89:33-39.

26. Scattoni V, Zlotta A, Montironi R, Schulman C, Rigatti P, Montorsi F. Extended and saturation prostatic biopsy in the diagnosis and characterisation of prostate cancer: a critical analysis of the literature. Eur Urol. 2007;52:1309-1322.

*27. Guichard G, Larrré S, Gallina A, Lazar A, Faucon H, Chemama S, et al. Extended 21-sample needle biopsy protocol for diagnosis of prostate cancer in 1000 consecutive patients. Eur Urol. 2007;52:430-435. 
28. Campos-Fernandes JL, Bastien L, Nicolaiew N, Robert G, Terry S, Vacherot F, et al. Prostate cancer detection rate in patients with repeated extended 21-Sample needle biopsy. Eur Urol. 2009;55:600-609.

29. Ashley RA, Inman BA, Routh JC, Mynderse LA, Gettman MT, Blute ML. Reassessing the diagnostic yield of saturation biopsy of the prostate. Eur Urol. 2008;53:976-983.

30. Djavan B, Ravery V, Zlotta A, Dobronski P, Dobrovits M, Fakhari M, et al. Prospective evaluation of prostate cancer detected on biopsies 1, 2, 3 and 4: when should we stop? J Urol. 2001;166:16791683.

*31. Djavan B, Remzi M, Schulman C, Marberger M, Zlotta A. Repeat prostate biopsy: who, how and when? A review. Eur Urol. 2002;42:93-103.

32. Mitterberger M, Pelzer A, Colleselli D, Bartsch G, Strasser H, Pallwein L, et al. Contrast-enhanced ultrasound for diagnosis of prostate cancer and kidney lesions. Eur J Radiol. 2007;64:231-238.

33. Tang J, Yang JC, Luo Y, Li J, Li Y, Shi H. Enhancement characteristics of benign and malignant focal peripheral nodules in the peripheral zone of the prostate gland studied using contrastenhanced transrectal ultrasound. Clin Radiol. 2008;63:1086-1091.

34. Wink M, Frauscher F, Cosgrove D, Chapelon JY, Palwein L, Mitterberger M, et al. Contrast-enhanced ultrasound and prostate cancer; a multicentre European research coordination project. Eur Urol. 2008;54:982-992.

35. Salomon G, Köllerman J, Thederan I, Chun FKH, Budäus L, Schlomm T, et al. Evaluation of prostate cancer detection with ultrasound real-time elastography: a comparison with step section pathological analysis after radical prostatectomy. Eur Urol. 2008;54:1354-1362.

36. Pallwein L, Aigner F, Faschingbauer R, Pallwein E, Pinggera G, Bartsch G, et al. Prostate cancer diagnosis: value of real-time elastography. Abdom Imaging. 2008;33:729-735.

37. Sciarra A, Salciccia S, Panebianco V. Proton spectroscopic and dynamic contrast-enhanced magnetic resonance: a modern approach in prostate cancer imaging. Eur Urol. 2008;54:485-488.

38. Braeckman J, Autier P, Garbar C, Pipeleers Marichal M, Soviany C, Nir R, Nir D, et al. Computer-aided ultrasonography (histo-scanning): a novel technology for locating and characterizing prostate cancer. GJU Int. 2007;101:293-298.

39. Marks LS, Fradet Y, Deras IL, Blase A, Mathis J, Aubin SM, et al. PCA3 Molecular urine assay for prostate cancer in men undergoing repeat biopsy. Urology. 2007;69:532-535.

40. Haese A, de la Taille A, van Poppel H, Marberger M, Stenzl A, Mulders PF, et al. Clinical utility of the PCA3 urine assay in European men scheduled for repeat biopsy. Eur Urol. 2008;54:1081-1088.

*41. Brawer MK, Lin DW, Williford WO, Jones K, Lepor H: Effect of finasteride and/or terazosin on serum PSA: results of VA cooperative study \#359. Prostate. 1999;39:234-239.

42. Thompson IM, Chi C, Ankerst DP, Goodman PJ, Tangen CM, Lippman SM, et al. Effect of finasteride on the sensitivity of PSA for detecting prostate cancer. J Natl Cancer Inst. 2006;98:1128-1133.

43. Andriole GL, Guess HA, Epstein JI, Wise H, Kadmon D, Crawford ED, et al. Treatment with finasteride preserves usefulness of prostate-specific antigen in the detection of prostate cancer: results of a randomized, double-blind, placebo-controlled clinical trial. PLESS Study Group. Proscar Long-term Efficacy and Safety Study. Urology. 1998;52:195-202.

44. Andriole GL, Marberger M, Roehrborn CG. Clinical usefulness of serum prostate specific antigen for the detection of prostate cancer is preserved in men receiving the dual 5alpha-reductase inhibitor dutasteride. J Urol. 2006;175:1657-1662.

45. Pannek J, Marks LS, Pearson JD, Rittenhouse HG, Chan DW, Shery ED, et al. Influence of finasteride on free and total serum prostate specific antigen levels in men with benign prostatic hyperplasia. J Urol. 1998;159:449-453.

46. Etzioni RD, Howlader N, Shaw PA, Ankerst DP, Penson DF, Goodman PJ, et al. Long-term effects of finasteride on prostate specific antigen levels: results from the prostate cancer prevention trial. $\mathrm{J}$ Urol. 2005; 174:877-881.

47. Oesterling JE, Roy J, Agha A, Shown T, Krarup T, Johansen T, et al. Biologic variability of prostatespecific antigen and its usefulness as a marker for prostate cancer: effects of finasteride. The Finasteride PSA Study Group. Urology. 1997;50:1318.

48. Moser PL, Brunner A, Horninger W, Bartsch G, Mikuz G. Correlation between inflammation cells ( $\mathrm{T}$ and B lymphocytes, macrophages) in prostate biopsies and elevated PSA levels in a PSA screening population. Urology. 2002;59:68-72.

49. Kefi A, Koseoglu H, Celebi I, Yorukoglu K, Esen A. Relation between acute urinary retention, chronic prostatic inflammation and accompanying elevated prostate-specific antigen. Scand J Urol Nephrol. 2006;40:155-160.

50. Morote J, Lopez M, Encabo G, de Torres IM. Effect of inflammation and benign prostatic enlargement on total and percent free serum prostatic specific antigen. Eur. Urol. 2000;37:537-540.

51. Nickel JC, Young DI, Boag S. Asymptomatic inflammation and/or infection in benign prostatic hyperplasia. BJU Int. 1999;84:976-981.

52. Ornstein D, Rao G, Smith D, Andriole G. The 
impact of systematic prostate biopsy on prostate cancer incidence in men with symptomatic benign prostatic hyperplasia undergoing transurethral resection of the prostate. J Urol. 1997;157:880884.

53. Puppo P, Introini C, Calvi P, Naselli A. Role of transurethral resection of the prostate and biopsy of the peripheral zone in the same session after repeated negative biopsies in the diagnosis of prostate cancer. Eur Urol. 2006;49:873-878.

54. Marberger MJ, Andersen JT, Nickel JC, Malice MP, Gabriel M, Pappas F, et al. Prostate volume and serum prostate-specific antigen as predictors of acute urinary retention. Eur Urol 2000;38:563568.

55. Roehrborn CG, Boyle P, Gould AL, Waldstreicher J. Serum prostate-specific antigen as a predictor of prostate volume in men with benign prostatic hyperplasia. Urology. 1999;53:581-589.

56. Laniado ME, Ockrim JL, Marronaro A, Tubaro A, Carter SS. Serum prostate-specific antigen to predict the presence of bladder outlet obstruction in men with urinary symptoms. BJU Int. 2004;94:1283-1286.

57. Roehrborn C, McConnell J, Lieber M, Kaplan S, Geller J, Malek G, et al. Serum prostate-specific antigen concentration is a powerful predictor of acute urinary retention and need for surgery in men with clinical benign prostatic hyperplasia. Urology. 1999;53:473-480.

58. Roehrborn C, McConnell J, Bonilla J, Rosenblatt S, Hudson P, Malek G, et al. Serum prostate specific antigen is a strong predictor of future prostate growth in men with benign prostatic hyperplasia. J Urol. 2000;163:13-20.

**59. Mochtar CA, Kiemeney LALM, Laguna MP, van Riemsdijk MM, Barnett GS, Debruyne FMJ, et al. Prognostic role of prostate-specific antigen and prostate volume for the risk of invasive therapy in patients with benign prostatic hyperplasia initially managed with alpha1-blockers and watchful waiting. Urology. 2005;65:300-305.

**60. Reich O, Gratzke C, Stief CG. Techniques and long-term results of surgical procedures for $\mathrm{BPH}$. Eur Urol. 2006;49:970-978.

61. Merrill R, Wiggins C. Incidental detection of population-based prostate cancer incidence rates through transurethral resection of the prostate. Urol Oncol. 2002;7:213-219.

62. McNeal J, Redwine E, Freiha F, Stamey T. Zonal distribution of prostatic adenocarcinoma. Correlation with histologic pattern and direction of spread. Am J Surg Pathol.1988;12:897-906.

63. Rovner E, Schanne F, Malkowicz B, Wein A. Transurethral biopsy of the prostate for persistently elevated or increasing prostate specific antigen following multiple negative transrectal biopsies. J
Urol. 1997;158:138-142.

64. Bales G, Flynn R, Kynaston H, Golash A, Hart A, $\mathrm{Kim} \mathrm{H}$, et al. Role of transurethral biopsy sampling of the prostate to diagnose prostate cancer in men undergoing surgical intervention for benign prostatic hyperplasia. Tech Urol. 2000;6:201204.

65. Kitamura H, Masumori N, Tanuma Y, Yanase M, Itoh N, Takahashi A, et al. Does transurethral resection of the prostate facilitate detection of clinically significant prostate cancer that is missed with systematic sextant and transition zone biopsies? Int J Urol. 2002; 9:95-99.

66. Radhakrishnan S, Dorkin TJ, Sheikh N, Greene DR: Role of transition zone sampling by TURP in patients with raised PSA and multiple negative transrectal ultrasound-guided prostatic biopsies. Prostate Cancer Prostatic Dis. 2004;7:338-342.

**67. Zigeuner R, Schips L, Lipsky K, Auprich M, Salfellner M, Rehak P, et al: Detection of prostate cancer by TURP or open surgery in patients with previously negative transrectal prostate biopsies. Urology. 2003;62:883-887.

68. Philip J, Dutta Roy S, Scally J, Foster CS, Javlé P. Importance of TURP in diagnosing prostate cancer in men with multiple negative biopsies. Prostate. 64;2005:200-202.

69. van Renterghem K, Van Koeveringe G, van Kerrebroeck P. Rising PSA in patients with minor LUTS without evidence of prostatic carcinoma: a missing link? Int Urol Nephrol 2007;39:11071113.

70. Sacks SH, Aparicio SA, Bevan A, Oliver DO, Will EJ, Davison AM. Late renal failure due to prostatic outflow obstruction: a preventable disease. $\mathrm{Br}$ Med J. 1989;298:156-159.

71. Rule AD, Lieber MM, Jacobsen SJ. Is benign protatic hyperplasia a risk factor for chronic renal failure? J Urol. 2005;173:691-696.

72. van Renterghem K, Van Koeveringe G, Achten R, van Kerrebroeck P. Clinical relevance of transurethral resection of the prostate in "asymptomatic" patients with an elevated prostate-specific antigen level. Eur Urol 2007;52:819-826

73. van Renterghem K, Van Koeveringe G, Achten R, van Kerrebroeck P. Long-term clinical outcome of a diagnostic transurethral resection of the prostate in patients with an elevated prostate-specific antigen level and minor lower urinary tract symptoms. Urol Int. 2009;83:60-65.

74. van Renterghem K, Van Koeveringe G, Achten R, van Kerrebroeck P. Prospective study of the clinical outcome of a TURP in patients with an elevated prostate specific antigen level, minor lower urinary tract symptoms and proven bladder outlet obstruction. Eur Urol. 2008;54(6):1385-1392.

**75. van Renterghem K, Van Koeveringe G, Achten R, 
van Kerrebroeck P. A new algorithm in patients with elevated and/or rising prostate-specific antigen level, minor lower urinary tract symptoms and negative multisite prostate biopsies. Int Urol Nephrol. 2009 Jun 3 (Epub ahead of print).

76. Rodrigues P, Lucon A, Freire G, Arap S. Urodynamic pressure flow studies can predict the clinical outcome after transurethral prostatic resection. J Urol. 2001;165:499-502.

77. Hakenberg OW, Pinnock CB, Marshall VR. Preoperative urodynamic and symptom evaluation of patients undergoing transurethral prostatectomy: analysis of variables relevant for outcome. Br J Urol. 2003;91:375-379.

78. Seki N, Takei M, Yamaguchi A, Naito S. Analysis of prognostic factors regarding the outcome after a transurethral resection for symptomatic benign prostatic enlargement. Neurourol Urodyn. 2006;25:428-432.

79. Griffiths D, Höfner K, van Mastrigt R, Rollema HJ, Spangberg A, Gleason D. Standardization of terminology of lower urinary tract function: pressure-flow studies of voiding, urethral resistance, and urethral obstruction. Neurourol Urodyn. 1997; 16:1-18.

80. Colombo R, Naspro R, Salonia A, Montorsi F, Raber M, Suardi N, et al. Radical prostatectomy after previous prostate surgery: clinical and functional outcomes. J Urol. 2006;176:2459-2463.

81. Teillac P, Scarpa RM. Economic issues in the treatment of BPH. Eur Urol Suppl.. 2006;5:10181024.

82. Madesbacher S, Marszalek M, Lackner J, Berger P, Schatzl G. The long-term outcome of medical therapy for BPH. Eur Urol. 2007;51:1522-1533.

83. Saigal C, Joyce G. Economic costs of benign prostatic hyperplasia in the private sector. J Urol. 2005; 173:1309-1313.

84. Disantostefano R, Biddle A, Lavelle J. An evaluation of the economic costs and patient-related consequences of treatments for benign prostatic hyperplasia. BJU Int. 2006;97:1007-1016.

85. Madersbacher S, Lackner J, Brossner C, Rohlich M, Stancik I, Willinger M, et al. Reoperation, myocardial infarction and mortality after transurethral and open prostatectomy: a nation-wide, long term analysis of 23,123 cases. Eur Urol. 2005;47:499-504.

86. Müntener M, Aellig S, Küttel R, Gehrlach C, Hauri D, Strebel RT. Peri-operative morbidity and changes in symptom scores after transurethral prostatectomy in Switzerland: results of an independent assessment of outcome. BJU Int. 2006;98:381-383.

87. Wendt-Nordahl G, Bucher B, Häcker A, Knoll T, Alken P, Michel MS. Improvement in mortality and morbidity in transurethral resection of the prostate over 17 years in a single center. J Endourol. 2007;21:1081-1087.

88. Reich O, Gratzke C, Bachmann A, Seitz M, Schlenker B, Hermanek P, et al. Morbidity, mortality and early outcome of transurethral resection of the prostate: a prospective multicenter evaluation of 10,654 Patients. J Urol. 2008;180:246-249.

89. Rassweiler J, Teber D, Kuntz R, Hofmann R. Complications of transurethral resection of the prostate (TURP) - Incidence, management and prevention. Eur Urol. 2006;50:969-980. 